\title{
BOD SENSOR FOR WASTEWATER ANALYSIS - DESIGN AND CALIBRATION METHODS
}

\author{
Siiri Velling \\ Kaja Orupõld \\ Toomas Tenno \\ University of Tartu, Estonia
}

\begin{abstract}
Water quality monitoring is an important aspect of water management concerning to the pollution control. The removal of biodegradable organic substances is a very important aspect of evaluation the treatment efficiency in a wastewater treatment plant (WWTP). The amount of oxygen consumed by microorganisms to oxidise organic substances is characterised by biochemical oxygen demand (BOD), a widely used parameter in environmental analysis. The conventional method BOD determination is uneffective from the point of view of short-term in situ measurements or on-line monitoring because it measures the microorganisms endogenous and exogenous oxygen consumption over a period of 5 or 7 days. BOD biosensors provide much faster results thereby allowing the researches to overcome the weaknesses of the conventional method.
\end{abstract}

BOD sensor studied in present work is based on an amperometric oxygen sensor and an easily replaceable biomembrane of mixed culture of microorganisms. Biosensor output signal depends on the concentration of substrate that indicates the organic pollution of waters. Biosensor output signal is analysed classically according to the steady-state parameters and by the dynamic change of the response and also by the elaborated mathematical model. The calibration of biosensor by steady-state method is based on the change of biosensor response between an initial value and the stable end-point of the signal. The dynamic method of measurement is based on the determination of the maximum rate of change of output signal during experiment and the mathematical model enables us to find the time constants characterising the non-steady-state processes in BOD sensor. Studied biosensor can be used for the measurements of biochemical oxygen demand in the concentration range $10-90 \mathrm{mg} / \mathrm{L}$.

\section{KEYWORDS}

BOD sensor; Immobilised microorganisms; Biochemical oxygen demand; Calibration methods; Non-steady-state processes; 


\section{INTRODUCTION}

Determination of biodegradable organic substances in wastewater has significant importance on the environmental pollution control. Traditional analysis of biochemical oxygen demand (BOD) involves 5 to 7 days incubation period and is thus ineffective for operation of activated sludge processes in wastewater treatment plants or determination of the concentration of biodegradable organic pollutants in polluted waters. Because of the need for express method of BOD determination, biochemical oxygen demand (BOD) biosensors have been developed. A biosensor is defined as a self-contained integrated device capable of providing specific quantitative analytical information using a biological recognition element [i]. BOD sensors are easy to handle, portative and offer quick response. Last aspect is especially advantageous as BOD sensors enable to evaluate the value of biochemical oxygen demand in 60 minutes.

Mathematical modelling of biosensors response provides several calibration methods and also gives a link among the design, fabrication and measurement procedure of the device. Most frequently the biosensor output signal is analysed according to the stabilised initial and final values. It has been shown that the total oxygen uptake measurement needs 15 $20 \mathrm{~min}$ and the recovery time between measurements can be as long as 3-4 h [ii]. The alternative approach to the steady-state analysis of a biosensors response [iii, iv, v] is through the mathematical modelling of the dynamic behaviour of the output values. Dynamic method is usually preferred as it allows to carry out faster measurements. In this study, the calibration of the BOD sensor involves both methods and also the calculation of time constants describing the non-steady-state processes in a BOD sensor. For the dynamic change of the biosensor output signal a model of transfer processes expressing the exponential decay and lag time of response by a transfer function has been developed [vi]. The function is characterised by an inflection point at which the change of the output signal shows a maximum value and by a time constant. The values of all the named variables depend on the substrate concentration affording to calibrate the biosensor by several methods.

\section{BOD SENSOR DESIGN}

In present study the designed BOD sensor based on the diffusion-limited amperometric oxygen sensor (the Clark-type oxygen sensor) and a biomembrane. The biomembrane consisted of mixed culture of microorganisms immobilised in $2 \%$ suspension of agarose in phosphate buffer. The agarose gel membrane was fixed on the top of the oxygen sensor polymer membrane with a special holder (see Figure 1).

Microorganisms used in biosensor were separated from the suspension of activated sludge originated from the Tartu WWTP. To accomplish a stable microbial consortium and improve mechanic properties of the biomembrane, the mixed culture of microorganisms was feed in laboratory scale process with a synthetic wastewater [5]. The $\mathrm{BOD}_{7}$ of this synthetic wastewater was $150 \mathrm{mg} / \mathrm{L}$. The $\mathrm{BOD}_{7}$ of this solution was $220 \pm 10$ $\mathrm{mg} / \mathrm{L}$. 
KALMAR ECO-TECH'03

Bioremediation and Leachate Treatment

KALMAR, SWEDEN, November 25-27, 2003

The microbial consortium was adapted in this synthetic wastewater for 2 weeks, then 40 $\mathrm{ml}$ of suspension was centrifuged at $400 \mathrm{rpm}$ for $15 \mathrm{~min}$. The supernatant was discarded and the remainder was washed with destined water and phosphate buffer solution (7.04 $\mathrm{g} / \mathrm{L} \quad \mathrm{NaH}_{2} \mathrm{PO}_{4} \times \mathrm{H}_{2} \mathrm{O}$ and $17.55 \mathrm{~g} / \mathrm{L} \quad \mathrm{Na}_{2} \mathrm{HPO}_{4} \times 12 \mathrm{H}_{2} \mathrm{O}$ ) (pro analysi, Merck) and centrifuged again. The remaining microorganisms were suspended in $4 \mathrm{ml}$ of phosphate buffer and $1.5 \mathrm{ml}$ of the final mix was immobilised with agarose.

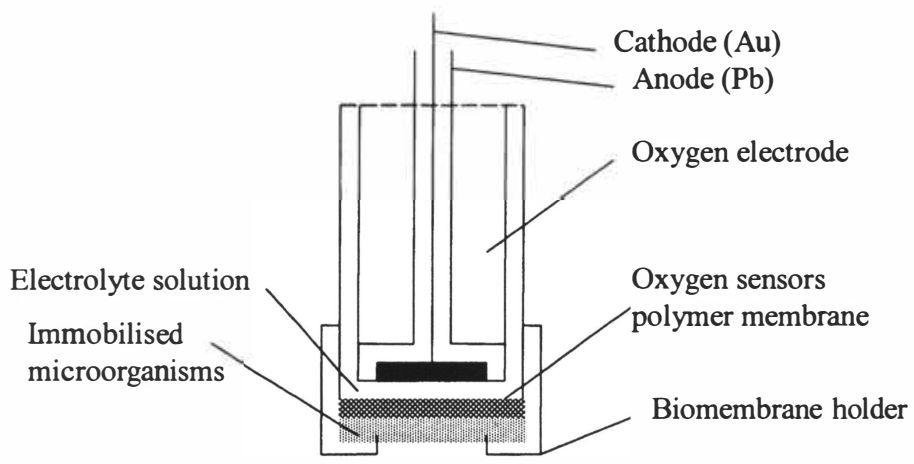

Figure 1. Schematic diagram of designed BOD sensor.

The $2 \%$ suspension of agarose (ultra pure, Sigma) in phosphate buffer was heated to the boiling temperature, then cooled down to $45^{\circ} \mathrm{C}$, and $1.5 \mathrm{ml}$ of prepared microorganisms suspension was added to it. The resulting mixture was spread on the polymer net of a particular thickness $(0.5 \mathrm{~cm})$ which, in order to gain a certain and even thickness of the layer containing microorganisms, was then placed between two glass plates until the formation of a persistent layer of gel. The microbial membrane was kept for 5 days in phosphate buffer to reach stable conditions. For the calibration of constructed biosensor the solution of D-glucose $(150 \mathrm{mg} / \mathrm{L})$ and L-glutamic acid $(150 \mathrm{mg} / \mathrm{L})(\mathrm{GGA})$ was used. The $\mathrm{BOD}_{7}$ of this solution was $220 \pm 10 \mathrm{mg} / \mathrm{L}$.

The measurements were carried out at $25^{\circ} \mathrm{C}$ in the thermostated measuring cell under continuous mixing with a magnetic stirrer and saturation of air oxygen with a microcompressor. The substrate solutions were added to the air-saturated test media after the stabilized steady-state output of the biosensor had been obtained. Sensor output signal was registered in a time interval of $1 \mathrm{~s}$.

\section{CALIBRATION METHODS OF A BOD SENSOR}

The calibration of constructed BOD sensor was performed by three different variables characterising the measurement process. The determination of the difference between the change of stabilised initial and final values of the biosensor response is a known method 
KALMAR ECO-TECH'03

Bioremediation and Leachate Treatment

KALMAR, SWEDEN, November 25-27, 2003

of biosensor calibration. Also dynamic method of measurements, that offers much faster response is covered in several studies [3-5, vii, viii]. The determination of time constants for calibration of BOD sensor that is based analogously to dynamic method on the course of output signal has been studied in the frames of this paper.

\subsection{Calibration of BOD sensor by the change of steady-state values of response and by dynamic method}

The steady-state response in the beginning of measurements shows the endogenous respiration of immobilised microorganisms. In a continuously mixed system dissolved oxygen diffuses from the aerated phosphate buffer to the biomembrane, where part of the oxygen is consumed by the microorganisms (see Figure 2). The remainder diffuses through the oxygen sensors polymer membrane and is detected by the oxygen electrode.

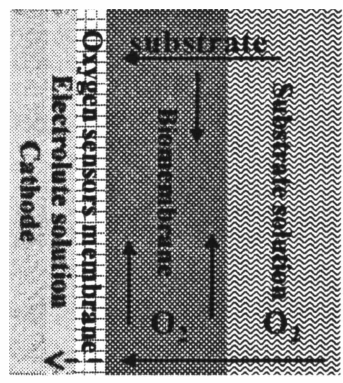

Figure 2. Schematic diagram of characteristic processes of substrate and oxygen in a BOD sensor.

When the substrate is added to the system, it diffuses also into the biomembrane and is assimilated by microorganisms, resulting in the increase of the amount of oxygen consumed. As a result, the concentration of oxygen on the polymer membrane of sensor decreases leading to a decrease in biosensor output signal.

A typical biosensor curve characterising the response of the BOD sensor for a sample of substrate with a BOD of $88 \mathrm{mg} / \mathrm{L}$ is depicted in Figure 3. The value of change of the stabilised initial and final values of response $(\Delta C)$ depends on the substrate concentration added after the stable initial signal of biosensor has been reached. The time for one measurement in steady-state regime takes approximately 12-15 minutes followed by 2050 min recovery time depending on substrate concentration. The study of non-steadystate response of biosensor output signal showed that to the maximum level of oxygen consumption by bacteria corresponds the maximum rate of change of output signal $\left(\mathrm{V}_{\mathrm{m}}\right)$ which value can be determined by the course of output signal. As it can be seen on the Figure 3, the dynamic method of measurements can be performed in a much shorter time. 
KALMAR ECO-TECH'03

Bioremediation and Leachate Treatment

KALMAR, SWEDEN, November 25-27, 2003

\subsection{Modelling of non-steady-state processes}

As a result of the further studies and mathematical modelling of non-steady-state processes in biosensor, a model describing the transfer processes in biosensor has been elaborated. The model is based on the main principles of working process of BOD sensors: ssubstrate from the test media, into which the sensitive are of the biosensor is immersed, is metabolised by microorganisms consuming oxygen in the biomembrane of the sensor. This process leads to the redistribution of oxygen fluxes and as a result, the concentration of oxygen decreases at oxygen sensors membrane leading to a decrease in the biosensor output signal. As in the non-steady-state regime the flux of oxygen towards the cathode is not proportional to the oxygen concentration in biomembrane layer, the biosensor response, lag time and processes in biomembrane are expressed by transfer function:

$$
h^{*}(t)=\exp \left(-\frac{t}{T_{s}}\right)-2 \sum_{n=1}^{\infty}(-1)^{n} \times \frac{T_{d}}{n^{2} T_{s}-T_{d}}\left[\exp \left(-\frac{t}{T_{s}}\right)-\exp \left(-n^{2} \frac{t}{T_{d}}\right)\right]
$$

where $T_{d}$ and $T_{s}$ are the time constants for the oxygen sensor and for the exponential decay of oxygen concentration at oxygen sensor membrane, respectively.

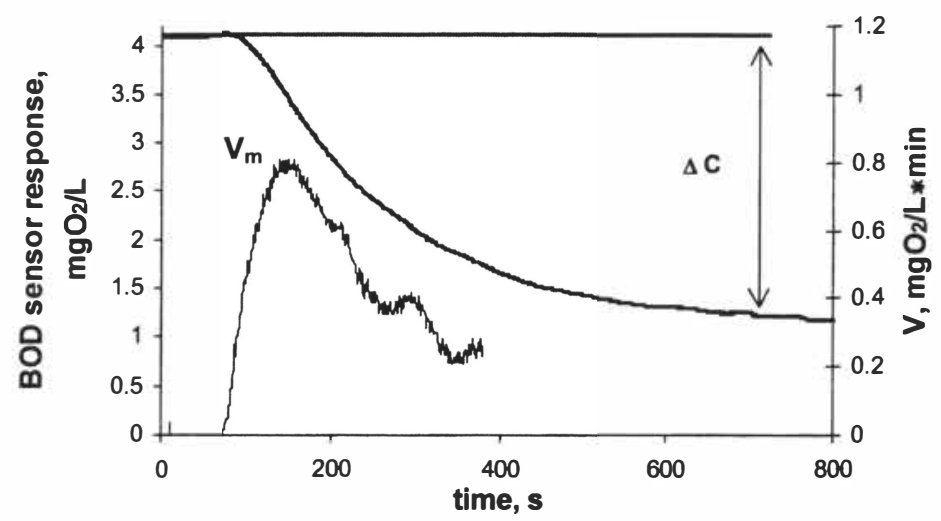

Figure 3. Analysis of output signal of a BOD sensor: $\square C$ - change of the stabilised initial and final values of response; $V$ - rate of change of sensors response; $V$ - maximum rate of change of the sensor output.

Transfer function of the oxygen sensor (see Equation 1) is characterised by time constant $\mathrm{T}_{\mathrm{s}}$ which value shows the time needed to run all non-steady-state processes of biosensor with maximum velocity, ie all the processes, diffusion and assimilation of substrate and oxygen and its electrochemical detection would have the maximum rate. On the basis of 
KALMAR ECO-TECH'03

Bioremediation and Leachate Treatment

KALMAR, SWEDEN, November 25-27, 2003

this knowledge, it is obvious, that $\mathrm{T}_{\mathrm{s}}$ depends on the substrate concentration in the test media.

\section{RESULTS AND DISCUSSION}

The biosensor response in the calibration solution (GGA) was detectable in concentration range $10-90 \mathrm{mg} / \mathrm{l}$ in BOD units (see Figure 4). The sensors response was analysed according to the steady-state method, dynamic determination and with the herein developed model of transfer processes.

The dependencies of the change of stabilised response and dynamic values of biosensor output signal $v s$ BOD concentrations of calibration solutions were characterised by hyperbolic relationships (see Figure $5(a)$ and (b)). For the calibration, the linear parts of these dependencies can be employed.

The hyperbolic relationships are characterised by theoretical maximum values of described variables $\left(\Delta \mathrm{C}_{\max }\right.$ or $\left.\mathrm{V}_{\max }\right)$ and by half-saturation coefficients that introduce the value of substrate concentration at which the variable under study has reached half of its maximum value. For the change of stabilised response and dynamic values of BOD sensor output signal the half-saturation coefficients are $18 \mathrm{mg} / \mathrm{L}$ and $27 \mathrm{mg} / \mathrm{L}$, respectively. Different values of half saturation coefficients can be explained by the various factors affecting the calibration methods. The change between stabilised initial and final value of output signal depends mostly on the number, viability and respiration rate of microorganisms. Determination of dynamic variables is influenced also by several other factors: the characteristics and concentration of immobilisation material, the thickness of biomembrane and the rate of substrate and oxygen diffusion.

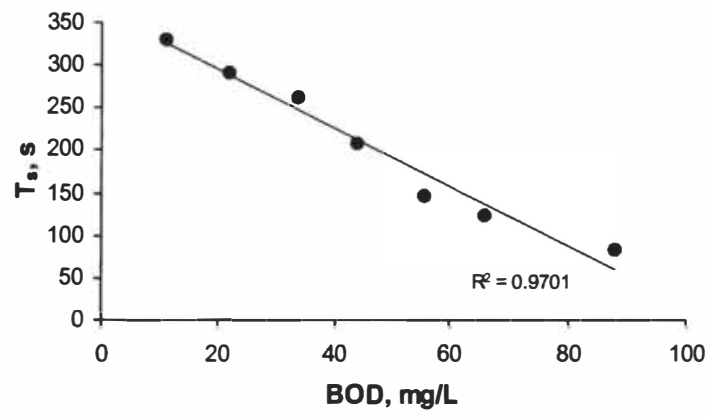

Figure 4. Normalised response of BOD sensor in calibration solution at various concentrations

The method of analysing the course of output signal of a biosensor thus provides a much broader measuring range and also shorter response and regeneration times. On the basis of these observations it is obvious that the method of using the maximum rate of change is more advantegeous for the practical applications of biosensors. 
(a)

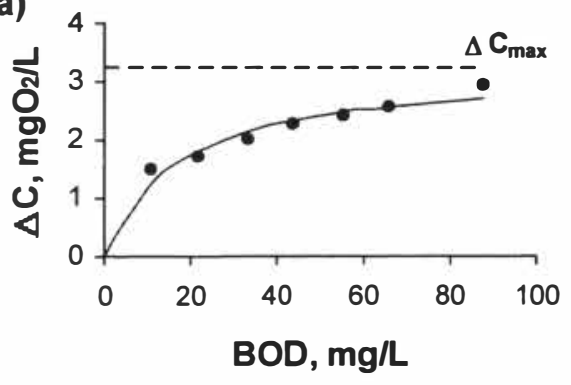

(b)

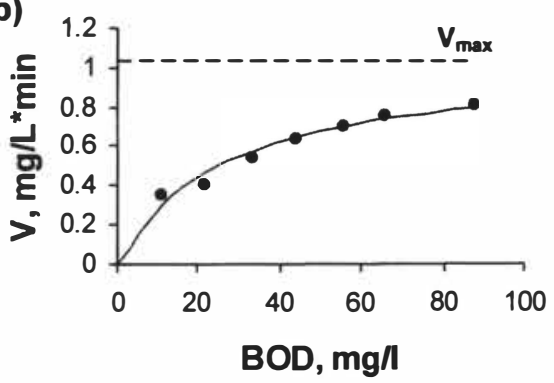

Figure 5. Dependence of the change of steady-state values $(\Delta C)(a)$ and the maximum rate of change $\left(V_{m}\right)$ (b) of biosensors response on substrate concentration. The solid lines correspond to the theoretical curve obtained by the least squares curve fitting.

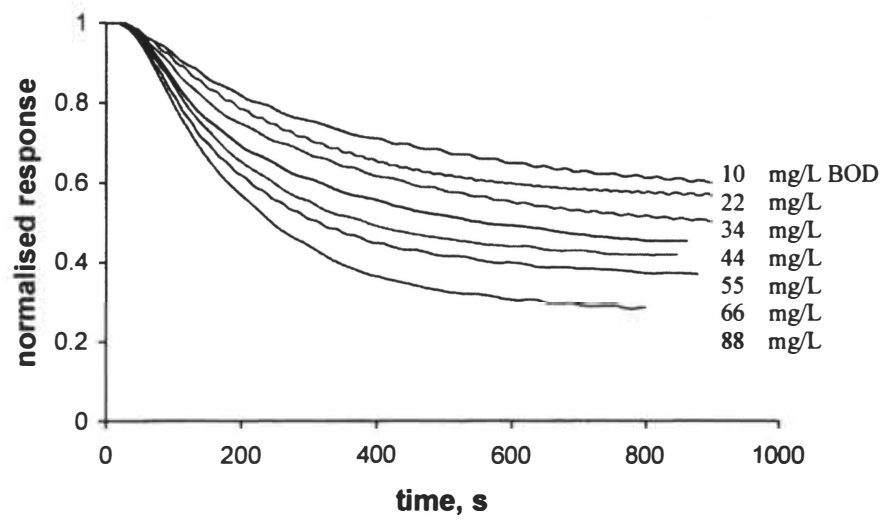

Figure 6. Dependence of time constant $T_{s}$ on substrate concentration.

Mathematical modelling of the response of studied BOD sensor enabled researches to work out a new calibration method that is based on non-steady-state processes in biosensor. The course of biosensor output signal can be characterised by time constants $T_{d}$ and $T_{s}$, that show the time lag and exponential decay of oxygen concentration at oxygen sensor membrane, respectively. The value of $T_{d}$, that depends on the immobilisation matrix and thickness of the biomembrane should not vary much in the case of similar experimental conditions. The average value of $T_{d}$ for constructed BOD sensor was $38 \pm 5 \mathrm{~s}$. The value of time constant $\mathrm{T}_{\mathrm{s}}$, calculated according to the formula 1 , depends on the substrate concentration as the increase of substrate consentration in test 
KALMAR ECO-TECH'03

Bioremediation and Leachate Treatment

KALMAR, SWEDEN, November 25-27, 2003

media causes the rise of oxygen consumption and therefore the amount of oxygen reaching the sensor membrane and oxygen electrode decreases. Linear dependence of $T_{s}$ vs calibration solution concentrations (see Figure 6) can be used for BOD sensor calibration.

\section{CONCLUSIONS}

The research confirmed that immobilised activated sludge based biosensor is a suitable tool for biochemical oxygen demand measurements and has good perspectives for quick and easy BOD measurements. Studied BOD sensor is sensible on organic substrate during 5-7 days, therefore easily replaceable biomembranes used in the study are preferred.

Designed BOD sensor is based on an amperometric oxygen sensor and biomembrane that consists of immobilised mixed culture of microorganisms. The calibration of BOD sensor involves several methods. Dynamic method of calibration, that shows the maximum rate of change of output signal widens the concentration range to be measured and fastens the measurement procedure compared to the method based on the change between stabilised initial and final response of BOD sensor. A new approach in the calibration of BOD sensor is the determination of time constants characterising the non-steady-state processes in biosensor. Mathematical modelling of response showed that linear dependce of the time constant of the exponential decay of oxygen concentration at oxygen sensor membrane $\left(T_{s}\right)$ on substrate concentration can be used for calibration of designed BOD sensor.

\section{REFERENCES}

[i] Thévenot, R. D., Toth, K., Durst, A. D. Wilson G. S., 2001. Electrochemical biosensors: recommended definitions and classification. Biosens. Bioelectr. 16, 121-131. DO1: https://doi.org/10.1016/S0956-5663(01)00115-4

[ii] Tan, T. C., Li, F., Neoh, K G., Lee, Y. K., 1992. Microbial membrane-modified dissolved oxygen probe for rapid biochemical oxygen demand measurement. Sens. Act., B. 8, 167-172. DO1: https://doi.org/10.1016/0925-4005(92)80175-W

[iii] Quian, Z., Tan, T. C., 1998. A model for multicomponent biosensing and its application to a dead cell-based BOD biosensor. Chem. Eng. Sci. 53, 3281-3294.

DO1: https://doi.org/10.1016/S0009-2509(98)00141-9

[iv] Li, F., Tan, T. C., Lee, Y. K., 1994. Effects of pre-conditioning and microbial composition on the sensing efficacy of a BOD biosensor. Biosens. Bioelect. 9, 197-205.

[v] Liu, J., Björnsson, L., Mattiasson, B., 2000. Immobilised activated sludge based biosensor for biochemical oxygen demand measurement. Biosens. Bioelect. 14, 883-893. DO1: https://doi.org/10.1016/S0956-5663(99)00064-0

[vi] Velling, S., Tammeveski, K., Mashirin, A., Tenno, T., 2002. Proc. Estonian Acad. Sci. Chem. 51, 34-48.

[vii] Chan, C., Lehmann, M., Chan, K., Chan, P., Chan, C., Gruendig, B., Kunze, G., Renneberg, R., 2000. Designing an amperometric thick-film microbial BOD sensor. Biosens. Bioelect. 15, 343-353. DO1: https://doi.org/10.1016/S0956-5663(00)00090-7

[viii]Tan, T. C., Li, F., Neoh, K. G., 1993. Measurement of BOD by initial rate of

response of a microbial sensor. Sens. Act., B. 10, 137-142. 